\title{
Estudio por GXRD de la formación de fases en metales de los grupos IV y $\mathrm{V}$ por implantación iónica de nitrógeno
}

\author{
J. A. GARCÍA', J. RIUS², R.J. RODRÍGUEZ1 \\ ${ }^{1}$ Centro de Ingeniería Avanzada de Superficies, AIN, 31191 Cordovilla-Pamplona \\ 2 Institut de Ciència de Materials de Barcelona, CSIC, Campus UAB, 08195 Bellaterra
}

\begin{abstract}
La implantación iónica de altas dosis de nitrógeno produce cambios significativos en la composición y estructura de las capas más externas de los materiales tratados. Estos cambios se reflejan en la modificación de las propiedades mecánicas superficiales, pudiendo conseguirse aumentos importantes de dureza y resistencia al desgaste. En el presente trabajo se presenta un resumen de los cambios en las propiedades mecánicas superficiales producidos por la implantación iónica de nitrógeno en los distintos metales de los grupos IV (Ti, Zr, Hf) y V (V, $\mathrm{Nb}, \mathrm{Ta}$ ), intentándose relacionar dichos cambios con las distintas fases formadas estudiadas mediante técnicas de difracción de rayos X con ángulo rasante (GXRD). La caracterización por GXRD pone de manifiesto el papel del nitrógeno como responsable de los cambios en la microdureza y de las propiedades tribológicas observadas.
\end{abstract}

Palabras clave: Implantación iónica, GXRD, Dureza, Tribología

\section{GXRD study of phase formation in metals of IV and V groups ion implanted with nitrogen}

High doses of nitrogen ion implantation introduce important modifications in the composition and microstructure of the most outer surface of the treated materials. Owing to this modifications, treated materials increase dramatically hardness and wear resistance. In this paper the changes in hardness and in the tribological properties of the groups IV and V are summarized. Special emphasis is given to the relationships between these improvements and the results obtained from GXRD analysis. Moreover GXRD results are showing the role of nitrogen in the improvements of hardness and the tribological properties.

Keywords: Ion implantation, GXRD, Hardness, Tribology

\section{INTRODUCCIÓN}

Los metales y aleaciones de los grupos IV y V (titanio, circonio, hafnio, vanadio, tantalo y tungsteno) son ampliamente utilizados en distintas aplicaciones industriales. Por ejemplo, el titanio y sus aleaciones son empleadas ampliamente en el sector aeroespacial y biomédico, el circonio y el vanadio en aplicaciones nucleares $(1,2)$, y el tántalo y el tungsteno en aplicaciones que requieran resistencia a elevadas temperaturas. Aunque estos materiales poseen en general buenas propiedades de resistencia mecánica, ofrecen unas pobres propiedades tribológicas, sufriendo altos grados de desgaste y un rápido deterioro superficial. Recientemente se han publicado estudios sistemáticos que recogen las mejoras que produce la implantación ionica de nitrógeno en estos materiales, apuntando a la precipitación de nitruros como el principal responsable de estas mejoras $(3,5)$. En este artículo se resumen y comentan los efectos y las modificaciones producidos por la implantación de distintas dosis de nitrógeno atómico y molecular sobre los metales de los grupos IV y V, tratando de relacionar los cambios en las propiedades tribológicas con la precipitación de las distintas fases caracterizadas por GXRD.

\section{DETALLES EXPERIMENTALES.}

\subsection{Materiales y preparación.}

Se prepararon probetas de Titanio de $30 \mathrm{~mm}$ de diámetro a partir de barras de Ti GR.2 recocido. El resto de los materiales se preparó en probetas de $20 \mathrm{~mm}$ x $20 \mathrm{~mm}$ y $2 \mathrm{~mm}$ de espesor a partir de planchas de los distinto metales de 99.9 de pureza en estado recocido. Posteriormente las probetas recibieron un pulido a espejo con Alúmina de
0.05 micras, y se sumergieron en acetona en un baño de ultrasonidos durante 15 minutos.

\subsection{Tratamientos}

Las implantaciones se realizaron con un implantador de alta corriente del Centro de Ingeniería Avanzada de Superficies de AIN a una energía de $150 \mathrm{keV}$. Los parámetros más relevantes de las implantaciones se muestran en la Tabla I. La selección de estos parámetros se llevó a cabo tras el estudio de los diagramas de equilibrio de los sistemas Me-N (metal-nitrógeno), y tras realizar las simulaciones mediante los programas TRIM96 y PROFILE Code.

TABLA I: RESUMEN DE LOS PARÁMETROS DE IMPLANTACIÓN

\begin{tabular}{|c|c|c|c|c|}
\hline Sustrato & $\begin{array}{c}\text { Dosis } \\
\text { (ion/ } / \mathrm{cm}^{2} \text { ) }\end{array}$ & Ion & $\begin{array}{l}\text { Posición del pico de } \\
\text { implantación }(\mathrm{nm})\end{array}$ & $\begin{array}{c}\text { Rango } \\
\text { máximo }(\mathrm{nm})\end{array}$ \\
\hline \multirow{2}{*}{ Titanio } & $4 \mathrm{e} 17$ & $\mathrm{~N}+$ & 151 & 350 \\
\hline & $8 \mathrm{e} 17$ & $\mathrm{~N} 2+$ & 122 & 180 \\
\hline \multirow{2}{*}{ Circonio } & $4 \mathrm{e} 17$ & $\mathrm{~N}+$ & 212 & 400 \\
\hline & $8 \mathrm{e} 17$ & $\mathrm{~N} 2+$ & 165 & 240 \\
\hline \multirow{2}{*}{ Hafnio } & $4 \mathrm{e} 17$ & $\mathrm{~N}+$ & 274 & 400 \\
\hline & $8 \mathrm{e} 17$ & $\mathrm{~N} 2+$ & 116 & 240 \\
\hline \multirow{2}{*}{ Vanadio } & $1.25 \mathrm{e} 17$ & $\mathrm{~N}+$ & 228 & 352 \\
\hline & $3.5 \mathrm{e} 17$ & $\mathrm{~N} 2+$ & 105 & 210 \\
\hline \multirow{2}{*}{ Niobio } & $1.25 \mathrm{e} 17$ & $\mathrm{~N}+$ & 154 & 278 \\
\hline & $3.5 \mathrm{e} 17$ & $\mathrm{~N} 2+$ & 64 & 190 \\
\hline \multirow{3}{*}{ Tántalo } & $1.25 \mathrm{e} 17$ & $\mathrm{~N}+$ & 132 & 292 \\
\hline & $7 \mathrm{e} 17$ & $\mathrm{~N}+$ & 108 & 320 \\
\hline & $3.5 \mathrm{e} 17$ & $\mathrm{~N} 2+$ & 42 & 188 \\
\hline
\end{tabular}




\subsection{Caracterización}

La dureza Universal H.U. (dureza bajo carga con indentador Vickers) se ha medido con un Microdurómetro FICHERSCOPE H100 empleando una carga final de $2 \mathrm{mN}$. Los ensayos de fricción se han realizado utilizando un Tribómetro FALEX ISC-320PC, con una configuración Ball-on-disk, empleando bolas de $1 / 2$ " de acero $100 \mathrm{Cr} 6$ y de Co-WC. El coeficiente de desgaste se calculó midiendo el volumen perdido en las trazas de los ensayos de fricción mediante un perfilómetro interferométrico WYCO RST 500 (6). Las mediciones de GXRD se han realizado en un difractómetro de polvo BRUKER D5000 provisto del accesorio de capas finas consistente en rendijas Soller, monocromador secundario de grafito y detector de centelleo. Las condiciones instrumentales fueron: $45 \mathrm{KV}, 25 \mathrm{~mA}$, radiación $\mathrm{K} \alpha$ del $\mathrm{Cu}(\lambda=1.5406 \AA$ ). El ángulo de incidencia fijo del haz de rayos- $X$ sobre la superficie de la muestra es de $1.3^{\circ}$ para todas las muestras. El valor óptimo de este ángulo había sido determinado previamente.

\section{RESULTADOS}

\subsection{Propiedades mecánicas superficiales}

Como puede observarse en la figura $1(4,5)$, todos los metales aumentan su microdureza al ser implantados con nitrógeno, en mayor medida para las dosis de implantación altas que para las dosis más pequeñas. Los incrementos de dureza con las diferentes implantaciones van desde un factor 1.4 para la dosis $1.25 \times 10^{17} \mathrm{~N}^{+} / \mathrm{cm}^{2}$ implantada sobre vanadio, hasta más de un factor 2 para la dosis $8 \times 10^{17} \mathrm{~N}^{+} / \mathrm{cm}^{2}$ implantada sobre circonio. La tabla II muestra una recopilación de los resultados de los coeficientes de fricción y de desgaste para muestras implantadas en las mismas condiciones que las aquí estudiadas $(4,5)$. De la tabla II se desprende que en los metales del grupo IV (titanio, circonio y hafnio) la implantación de dosis bajas parece ser más efectiva para la disminución de la fricción y el aumento de la resistencia al desgaste (4). En estos casos es posible observar como para dosis altas de nitrógeno molecular el coeficiente de fricción aumenta desde valores próximos a 0.3 hasta valores propios de las muestras sin implantar. Sin embargo las muestras implantadas con dosis bajas muestran un coeficiente de fricción bajo durante todo el ensayo. Este hecho concuerda con el aumento de resistencia al desgaste que para muestras implantadas con dosis bajas fue prácticamente nulo (dada su escasa magnitud no fue posible medirlo empleando el método descrito en el apartado $2.3(6)$ ).

Como también recoge la taba II, para las muestras de los metales del grupo $\mathrm{V}$ los tres metales mostraron comportamientos muy diferentes (5). El coeficiente de fricción del vanadio no presenta diferencias claras entre la muestra sin implantar y la preparadas con diferentes dosis. En cambio su coeficiente de desgaste disminuye en casi dos ordenes de magnitud para la dosis de $3.5 \times 10^{17} \mathrm{~N}_{2}^{+} / \mathrm{cm}^{2}$. El Niobio reduce considerablemente el coeficiente de fricción para las muestras implantadas con alta dosis, pero no existen apenas diferencias en la resistencia al desgaste entre las muestras implantadas y las de referencia. Para el tántalo tanto la implantación a baja como a alta dosis muestran descensos en el coeficiente de fricción, pero no alteran los coeficientes de desgaste.

\subsection{XRD con ángulo rasante}

A continuación se presentan los resultados obtenidos por GXRD para las diferentes muestras metales:
TABLAII: RESUMEN DE LOS RESULTADOS DE LOS ENSAYOS DE FRICCIÓN Y DESGASTE

\begin{tabular}{|c|c|c|c|c|c|}
\hline \multirow{2}{*}{ Muestra } & \multirow{2}{*}{$\begin{array}{c}\text { Dosis } \\
\text { (iones } / \mathrm{cm}^{2} \text { ) }\end{array}$} & \multicolumn{3}{|c|}{ Coeficiente de fricción } & \multirow{2}{*}{$\begin{array}{l}\begin{array}{l}\text { Coeficiente } \\
\text { desgaste } \\
\left(\mathrm{N}^{-1} \mathbf{m}^{2}\right)\end{array} \\
\text { Bola: WC } \\
\text { Hr: } 50 \%\end{array}$} \\
\hline & & $\begin{array}{l}\text { Bola: } 100 \mathrm{Cr} 6 \\
\text { Hr: } 30 \%\end{array}$ & $\begin{array}{l}\text { Bola: } 100 \mathrm{Cr} 6 \\
\text { Hr: } 50 \%\end{array}$ & $\begin{array}{l}\text { Bola 100Cr6 } \\
\text { Hr: } 70 \%\end{array}$ & \\
\hline \multirow{3}{*}{$\begin{array}{l}\text { Titanio } \\
\text { (4) }\end{array}$} & Referencia & $\rightarrow 0,86$ & $\rightarrow 0,8$ & $\rightarrow 0,84$ & $1,10 \mathrm{E}-12$ \\
\hline & $4 \mathrm{E} 17 \mathrm{~N}^{+}$ & 0,30 & 0,30 & $0,26-0,30$ & --- \\
\hline & $8 \mathrm{E} 17 \mathrm{~N}_{2}^{+}$ & $0,30 \rightarrow 0,86$ & $0,30 \rightarrow 0,8$ & $0,30 \rightarrow 0,70$ & $9,71 \mathrm{E}-13$ \\
\hline \multirow{3}{*}{$\begin{array}{l}\text { Circonio } \\
\text { (4) }\end{array}$} & Referencia & $\rightarrow 0,8$ & $\rightarrow 0,6$ & $\rightarrow 0,84$ & $1,68 \mathrm{E}-12$ \\
\hline & $4 \mathrm{E} 17 \mathrm{~N}^{+}$ & 0,31 & 0,20 & $0,26-0,30$ & --- \\
\hline & $8 \mathrm{E} 17 \mathrm{~N}_{2}^{+}$ & $0,2 \rightarrow 0,8$ & $0,20 \rightarrow 0,7$ & $0,30 \rightarrow 0,70$ & $4,12 \mathrm{E}-13$ \\
\hline \multirow{3}{*}{$\begin{array}{l}\text { Hafnio } \\
\text { (4) }\end{array}$} & Referencia & $\rightarrow 0,8$ & $\rightarrow 0,86$ & $\rightarrow 0,84$ & $1,69 \mathrm{E}-12$ \\
\hline & $4 \mathrm{E} 17 \mathrm{~N}^{+}$ & 0,30 & 0,30 & $0,26-0,30$ & $8,62 \mathrm{E}-15$ \\
\hline & $8 \mathrm{E} 17 \mathrm{~N}_{2}^{+}$ & 0,48 & $0,30 \rightarrow 0,86$ & $0,30 \rightarrow 0,70$ & 3,12 E-13 \\
\hline \multirow{3}{*}{$\begin{array}{l}\text { Vanadio } \\
\text { (5) }\end{array}$} & Referencia & $0.6-0.8$ & $0.6-0.8$ & $0.5-0.6$ & $1.57 \mathrm{e}-13$ \\
\hline & $1.25 \mathrm{E} 17 \mathrm{~N}^{+}$ & $0.6-0.8$ & $0.2 \rightarrow 0.8$ & $0.5-0.6$ & $1.69 \mathrm{e}-13$ \\
\hline & $3.5 \mathrm{E} 17 \mathrm{~N}_{2}^{+}$ & 0.5 & $0.7-0.8$ & 0.5 & $3.44 \mathrm{e}-15$ \\
\hline \multirow{3}{*}{$\begin{array}{l}\text { Niobio } \\
\text { (5) }\end{array}$} & Referencia & 0.8 & $0.7-0.9$ & $0.8-.07$ & $4.90 \mathrm{e}-16$ \\
\hline & $1.25 \mathrm{E} 17 \mathrm{~N}^{+}$ & 0.8 & $0.3 \rightarrow 0.7$ & 0.8 & $4.39 \mathrm{e}-16$ \\
\hline & $3.5 \mathrm{E} 17 \mathrm{~N}_{2}^{+}$ & $0.2 \rightarrow 0.35$ & $0.2 \rightarrow 0.5$ & 0.2 & $4.55 \mathrm{e}-16$ \\
\hline \multirow{3}{*}{$\begin{array}{l}\text { Tántalo } \\
\text { (5) }\end{array}$} & Referencia & $0.7-0.6$ & $0.8-1$ & $0.8 \rightarrow 0.5$ & $1.82 \mathrm{e}-16$ \\
\hline & $1.25 \mathrm{E} 17 \mathrm{~N}^{+}$ & 0.2 & 0.2 & 0.45 & $1.67 \mathrm{e}-16$ \\
\hline & $3.5 \mathrm{E} 17 \mathrm{~N}_{2}^{+}$ & $0.2 \rightarrow 0.5$ & $\rightarrow 0.8$ & $0.2 \rightarrow 0.45$ & $2.35 \mathrm{e}-16$ \\
\hline
\end{tabular}

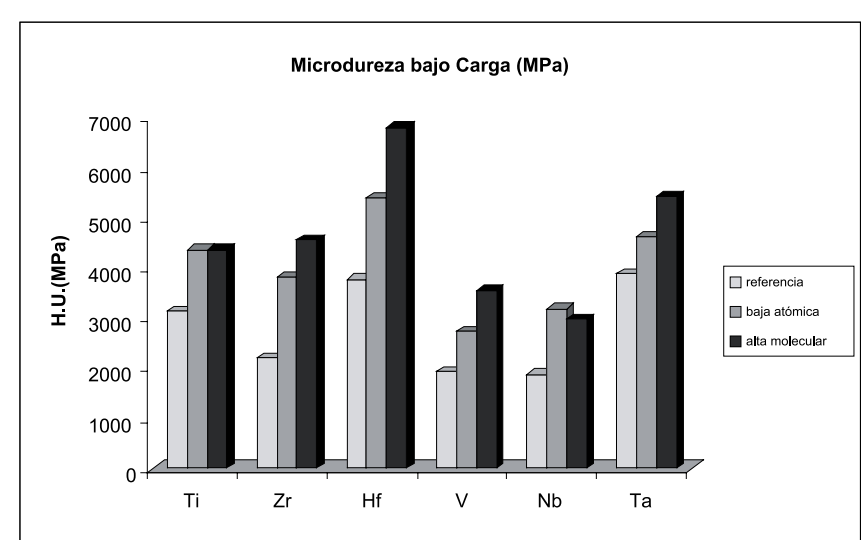

Figura 1: Resumen de microdureza para las distintas muestras implantadas y para las muestras de referencia

Titanio (Fig. 2a).- En esta figura se muestra el diagrama de difracción del titanio sin implantar, y de la muestra implantadas con $4 \times 10^{17}$ $\mathrm{N}^{+} / \mathrm{cm}^{2}$. En este último se aprecia un corrimiento hacia ángulos de difracción menores debido a la suma de la contribución del soporte (titanio sin implantar) más una fase $\left(\approx \mathrm{TiN}_{03}\right)$ con la celda cristalina ligeramente mayor que la del soporte (7). En el diagrama de la muestra implantada a $8 \times 10^{17} \mathrm{~N}_{2}{ }^{+}$aparecen los picos (111) y (200) de la fase cúbica centrada en las caras TiN (8). Con menor intensidad, pero también de manera clara se observan los picos (220) y (311). En cualquier caso y de acuerdo con otros trabajos (9-11), el TiN crece preferencialmente en las orientaciones (111) y (200).

Circonio (Fig. 2b).- En las muestras implantadas con $4 \times 10^{17}$ de nitrógeno atómico no aparecen nuevos picos que puedan ser asociados a precipitados de nitruros de circonio, apareciendo solamente las reflexiones del metal (12) aunque si puede apreciarse un cierto desplazamiento de los picos hacia ángulos de difracción menores debido a la distorsión de la celda por la incorporación de nitrógeno intersticial. En el diagrama de la muestra implantada con $8 \mathrm{E} 17 \mathrm{~N}_{2}+/ \mathrm{cm}^{2}$ es también posible apreciar la formación de precipitados $\mathrm{ZrN}$ con orientación (111) y (200) (13), de acuerdo con los trabajos de Miyagawa et al. y Etoh et al. (14-18). A diferencia de los trabajos anteriores en la figura 2.b no 

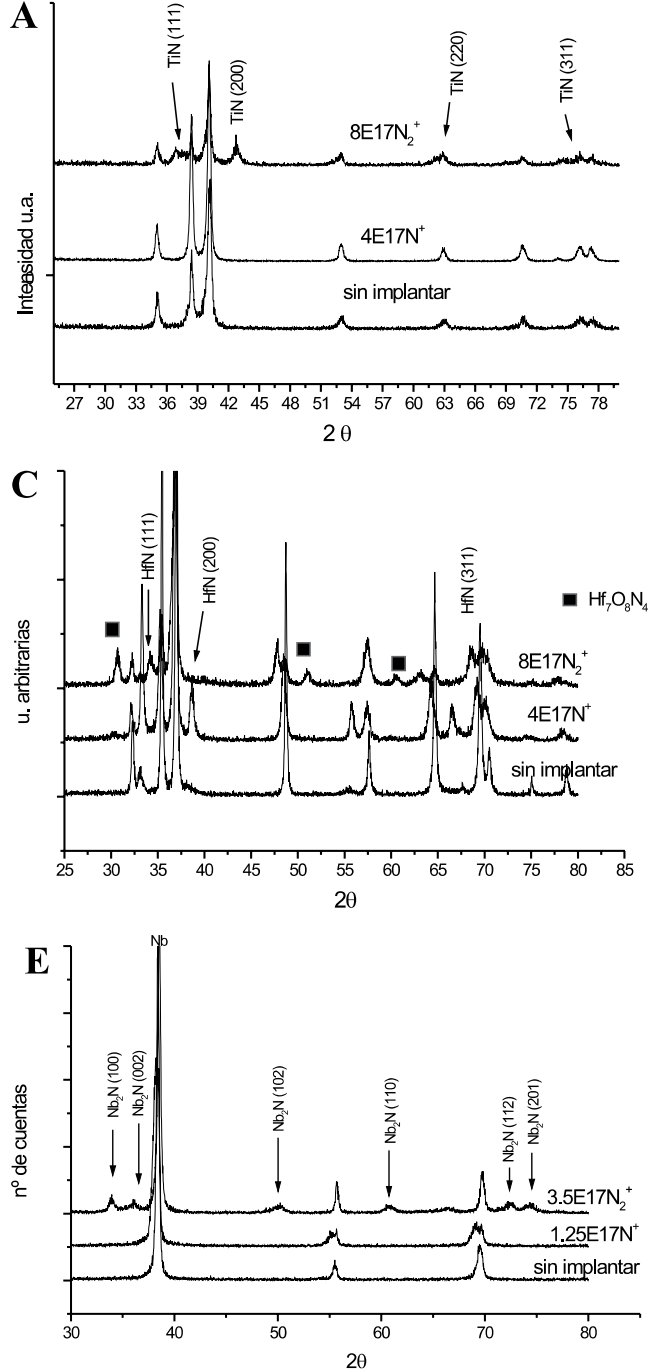

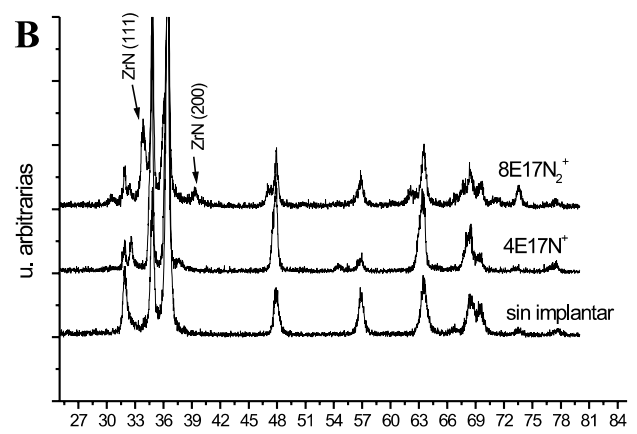

$2 \theta$
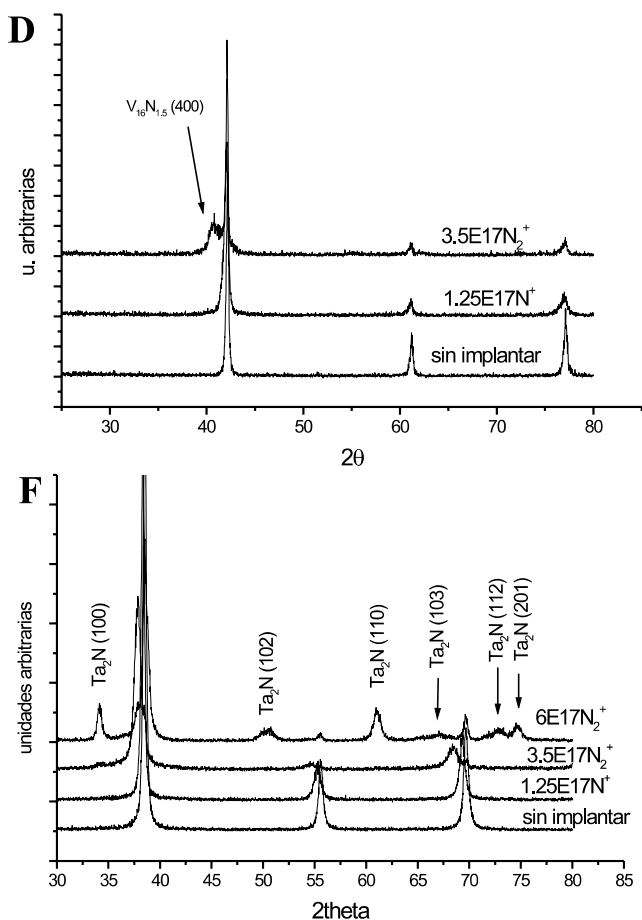

Figura 2: Espectros XRD para las muestras de titanio(A), las muestras de circonio(B), las muestras de hafnio (C), las muestras de vanadio (D), las muestras de niobio (E) y las muestras de tántalo $(\mathrm{F})$.

se observan los picos (220) del $\mathrm{ZrN}$, apareciendo en cambio una serie de reflexiones sin identificar $\left(2 \theta=30.55 ; 47.06\right.$ y $\left.62.09^{\circ}\right)$ que podrían estar asociadas a precipitados de óxidos de circonio o de algún tipo de compuesto ternario $\mathrm{Zr}(\mathrm{N}, \mathrm{O})$.

Hafnio (Fig. 2c).- En los diagramas de difracción de las muestras de hafnio de referencia e implantadas con $4 \times 10^{17} \mathrm{~N}^{+} / \mathrm{cm}^{2}$ no se observa con claridad la aparición de picos correspondientes a nuevas fases, y si parece existir un cierto desplazamiento de los picos del metal base (19) hacia ángulos de difracción menores. Por otro lado en el diagrama de difracción de las muestra implantada con $8 \times 10^{17} \mathrm{~N}_{2}^{+} / \mathrm{cm}^{2}$ y en la muestra de referencia, es posible apreciar los picos del nitruro de hafnio orientado preferencialmente en las direcciones (111); (200) y (311). En este diagrama también se observan los picos de un compuesto ternario, presumiblemente el oxinitruro $\mathrm{Hf}_{7} \mathrm{O}_{8} \mathrm{~N}_{4}(20)$.

Vanadio (Fig. 2d).- Para la muestra de vanadio implantada con $1.25 \times 10^{17} \mathrm{~N}^{+} / \mathrm{cm}^{2}$ se observa un desplazamiento de los picos de difracción hacia ángulos más pequeños, indicando la deformación de la celda centrada en el interior del vanadio por la presencia del nitrógeno en disolución intersticial en la fase $\alpha-\mathrm{V}(\mathrm{N})(21)$. Para la muestra implantada con $3.5 \times 10^{17} \mathrm{~N}_{2}^{+} / \mathrm{cm}^{2}$ no se observa el citado corrimiento, apareciendo un nuevo pico correspondiente a la reflexión (400) de la fase tretragonal de precipitados de $\mathrm{V}_{16} \mathrm{~N}_{1.5}$ (21) En el caso del vanadio en lugar de la fase hexagonal $\beta-V_{2} N_{1-y}$ predicha por el diagrama de equilibrio para las distintas muestras aparece esta nueva fase metaestable. La existencia de esta fase intermedia entre la $\alpha-\mathrm{V}(\mathrm{N})$ y la hexagonal $\beta-V_{2} \mathrm{~N}_{1-\mathrm{y}}$ esta de acuerdo con resultados recientes encontrados en el sistema V-N (22).

Niobio (Fig. 2e).- Tras la implantación de $1.25 \times 10^{17} \mathrm{~N}^{+} / \mathrm{cm}^{2}$, el diagrama de difracción correspondiente no muestra la aparición de nuevas fases, como predecían las consideraciones termodinámicas. Se observa simplemente un corrimiento hacia ángulos más pequeños debido a la deformación de la red por la incorporación de nitrógeno en la matriz de niobio, en concordancia con otros trabajos (23). Para las muestras implantadas con $3.5 \times 10^{17} \mathrm{~N}_{2}^{+} / \mathrm{cm}^{2}$ aparecen los picos de difracción de la fase hexagonal $\mathrm{Nb}_{2} \mathrm{~N}$ (24) de acuerdo con los perfiles experimentales, con las simulaciones y con los resultados de otros trabajos (23), pero no se observan ni los picos de la fase tetragonal $\mathrm{Nb}_{3} \mathrm{~N}_{4}$ tal y como se esperaba teniendo en cuenta los perfiles teóricos, ni los de la fase cúbica $\mathrm{NbN}(25)$.

Tántalo (Fig. 2f).- En la figura se muestran los diagramas de difracción de las probetas sin implantar y de las dos implantaciones con nitrógeno atómico. A pesar de los cálculos termodinámicos que 
predecían la presencia de la fase hexagonal primitiva $\mathrm{Ta}_{2} \mathrm{~N}$, en ambas implantaciones, solamente se observa el corrimiento de picos hacia angulos menores debido a la distorsión de la celda por la incorporación del nitrógeno (26). Para las muestras implantadas con $3.5 \times 10^{17} \mathrm{~N}_{2}{ }^{+}$/ $\mathrm{cm}^{2}$ los diagramas de difracción revelan la existencia de la fase hexagonal $\mathrm{Ta}_{2} \mathrm{~N}(27)$, como puede observarse en la figura $2 \mathrm{f}$, y de acuerdo con Yadav et al. (28), aunque a diferencia de estos últimos donde se informaba sobre la presencia de una fase intermedia $\mathrm{TaN}_{0.8}$ y la fase $\mathrm{Ta}_{2} \mathrm{~N}$ para dosis mayores de $5 \times 10^{16} \mathrm{~N}_{2}^{+} / \mathrm{cm}^{2}$, en nuestros resultados no se encuentra la fase hexagonal $\mathrm{Ta}_{2} \mathrm{~N}$ para dosis inferiores s $3.5 \times 10^{17}$ $\mathrm{N}_{2}^{+} / \mathrm{cm}^{2}$.

\section{CONCLUSIONES}

En líneas generales los resultados del apartado anterior muestran que la microdureza de los distintos metales aumenta con la dosis implantada, a excepción del niobio en el que si parece alcanzar una dosis de saturación. Por otro lado otra tendencia general mostrada por los 6 metales es la parición de fases de nitruros para las dosis altas de implantación, mientras que para las dosis bajas de nitrógeno atómico el nitrógeno se encuentra disuelto intersticialmente en la matriz de los metales.

A pesar de estas tendencias comunes para los 6 metales, los elementos del grupo IV (Ti, Zr, Hf) y los del grupo $\mathrm{V}(\mathrm{V}, \mathrm{Nb}, \mathrm{Ta}$ ) mostraron diferentes propiedades tribológicas al ser implantados. Tanto el titanio como el circonio y hafnio mostraron importantes descensos del coeficiente de fricción y del coeficiente de desgaste para las dosis bajas de nitrógeno atómico, mientras que no aparecen claras diferencias en le caso de los metales del grupo $\mathrm{V}$ a excepción del vanadio que reduce su desgaste en casi un factor 2 para las muestras implantadas a alta dosis de nitrógeno molecular.

Teniendo en cuenta los anteriores resultados, los aumentos de dureza y resistencia al desgaste mostrado por algunos de los metales implantados no pueden atribuirse únicamente a la presencia de nitruros, sino que también debe tenerse en cuenta el efecto producido por la incorporación de nitrógeno de forma intersticial deformando la matriz metálica sobre las propiedades mecánicas superficiales.

Agradecimientos: Los autores desean expresar su agradecimiento a la CICYT por el apoyo recibido mediante el proyecto MAT 1012, así como al Gobierno de Navarra (Protocolo de colaboración AquitaniaNavarra) proyecto TRIBOTEC.

\section{BIBLIOGRAFÍA}

1. J. A. García, R. Sánchez, R. Martínez, A. Medrano, M. Rico, R. J. Rodríguez, M. Varela, I. Colera, D. Cáceres, I. Vergara, C. Ballesteros, E. Román and J. L. de Segovia "Surface mechanical effects of nitrogen ion implantation on vanadium and vanadium alloys" Surf. Coat. Tech. 158-159C 669-673(2002).
2. J. A. García, R. Rodríguez, R. Sánchez, R. Martínez, C. Ballesteros, M. Varela D. Cáceres, I. Vergara "Tribological study of vanadium based alloys ion implanted at low energy-high temperature". Vacuum 67 [3-4] 543-550 (2002).

3. J. A. García, A. Guette, A. Medrano, C. Labrugere, M. Rico, M. Lahaye, R. Sánchezand R. J. Rodríguez."Modificación del comportamiento tribológico de Circonio y Titanio mediante la implantación de Nitrógeno" Bol. Soc. Esp. Ceram. V. 39 [3], 337 (2000).

4. J. A. García, A. Guette, A. Medrano, C. Labrugere, M. Rico, M. Lahaye, R. Sánchez, R. Martínez and R. J. Rodríguez "Nitrogen ion implantation on Group IV metals: Chemical, structural and tribological study" Vacuum 64, 343 (2002).

5. J. A. García, R. J. Rodríguez, A. Medrano, R. Sánchez, M. Rico, R. Martínez, C. Labrugere, M. Lahaye, and A. Guette "Study of the tribological modifications induced by nitrogen implantation on group Va metals" Surf. Coat. Tech. 158-159C 653-657 (2002).

6. Standar test method for wear testing with a Pin-on-Disk Apparatus ASTM G99-95

7. Standard X-ray diffraction pattern ficha ICDD No. 41-1352

8. Standard X-ray diffraction pattern ficha ICDD No 38-1420

9. S. Fukumoto, H. Tsubakino, S. Inoue, L. Liu, M. Terasawa, T. Mitamura “Surface modification of titanium by nitrogen ion implantation" Mater. Sci. Eng. A263 205-209 (1999).

10. M. Guemmaz, A. Mosser, J.J. Grob, “Ion implantation processing of substoichiometric titanium nitrides and carbonitrides: chemical structural and micromechanical investigations" Appl. Phys. A64 407-415 (1997).

11. R. Hutchings "The Subsurface Microstructure of Nitrogen-implanted Metals" Mater.Sci. Eng. 69 129-138 (1985).

12. Standard X-ray diffraction pattern ficha ICDD:05-0665

13. Standard X-ray diffraction pattern ficha ICDD:35-753

14. S. Miyagawa, K. Saitoh, M. Ikeyama G. Massouras, Y. Miyagawa."Composition and structure of zirconium implanted with nitrogen at high fluence". Nucl. Instr. And Meth, B69 437-442 (1992).

15. S. Miyagawa , M. Ikeyama, K. Saitoh, S. Nakao, Y. Shakai, Y. Miyagawa."Behaviour of nitrogen implanted into $\mathrm{Zr}$ at high fluence". Nucl. Instr. And Meth, B80/81 480-484 (1993).

16. S. Miyagawa, M. Ikeyama, K. Saitoh, S. Nakao, H. Niwa, S. Tamemura, Y Miyagawa "Thermal behaviour of nitrogen implanted into zirconium" Surf Coat Technol. 66 245-249 (1994).

17. Miyagawa Y, Nakao S, Saitoh K, Ikeyama M, Tanemura S, Miyagawa S. Nucl Instr Method. 1995; B 106:170-173.

18. Y. Etoh, S. Shimada and H. Takahashi.. "Surface improvement of $\mathrm{Zr}$ and Zircaloy-2 by metal implantation" J. Nucl. Mater. 202 122-136. ( 1993)

19. Standard X-ray diffraction pattern ficha ICDD:38-1420

20. Standard X-ray diffraction pattern ficha ICDD:39-521

21. Standard X-ray diffraction pattern ficha ICDD:22-1058

22. Standard X-ray diffraction pattern ficha ICDD:71-1230

23. I. Galesic, C. Angelkort, H. Lewalter, A. Berendes, B.O. Kolbesen." Formation of Metal Nitrides by rapid Thermal Processing" Phys. Stat. Sol. A177 15-26 (2000)

24. Standard X-ray diffraction pattern ficha ICDD:75-1616

25. X. Junhua, G. Mingyuan, L. Geyang. "Microstructure and mechanical properties of polycrystaline NbN/ TaN superlattice films" J. Matter. Sci. 35 35353538 (2000).

26. Standard X-ray diffraction pattern ficha ICDD:25-1276

27. Standard X-ray diffraction pattern ficha ICDD:26-985

28. A.D. Yadav, s.K. Dubey, G.K. Gupta, T.K. Gundu Rau. "Structural and electrical properties of high dose nitrogen implanted tantalum" Rad. Eff. Def. Sold. 153 245-33 (2000)

Recibido: 1.2 .03

Aceptado: 30.11 .03 